\title{
Extramatrical mycelium and ectomycorrhizal community composition of Quercus pubescens in a Sub-Mediterranean stress- prone environment
}

\author{
Tanja Mrak $^{1 *}$, Nataša Šibanc ${ }^{1,2}$, Philip Brailey-Jones ${ }^{1}$, Ines Štraus ${ }^{1}$, Jožica Gričar ${ }^{3}$, Hojka \\ Kraigher $^{1}$ \\ ${ }^{1}$ Department of Forest Physiology and Genetics, Slovenian Forestry Institute, Večna pot 2, 1000 \\ Ljubljana, Slovenia, ${ }^{2}$ Department of Agronomy, University of Ljubljana, Biotechnical Faculty, \\ Jamnikarjeva 101, 1000 Ljubljana, Slovenia, ${ }^{3}$ Department of Forest Yield and Silviculture, Slovenian \\ Forestry Institute, Večna pot 2, 1000, Ljubljana, Slovenia \\ * Correspondence: \\ Tanja Mrak \\ tanja.mrak@gozdis.si
}

\section{Supplementary material}

\section{Supplementary Results}

Soil-inhabiting extramatrical mycelium communities

The presence of fungi assigned to the saprotroph-symbiotroph trophic mode was related to the detection of higher taxonomic ranges (e.g. families) that contain taxa with both trophic strategies. However, there was still a significant proportion of pure saprotrophs and unassigned taxa. Plot 1 and 2 had a significantly higher proportion of both pure symbiotrophs and saprotroph-symbiotrophs (Kruskall-Wallis SaprotrophSymbiotroph, $\chi 2=9.774$, $\mathrm{df}=2, \mathrm{P}=0.008$; Symbiotroph, $\chi 2=6.3581$, $\mathrm{df}=2, \mathrm{P}=0.0416$, Supplementary Figure 6). The number of taxa which were not able to be assigned to a trophic mode also significantly differed among plots (Kruskall-Wallis, $\chi 2=8.498, \mathrm{df}=2, \mathrm{P}=0.0143$ ). There were no observed statistical differences among any plots for other trophic modes (Kruskall-Wallis Pathotroph, $\chi 2$ $=4.748, \mathrm{df}=2, \mathrm{P}=0.0931$; Pathotroph-Saprotroph, $\chi 2=1.0876, \mathrm{df}=2, \mathrm{P}=0.5805$; PathotrophSymbiotroph, $\chi 2=0.5286, \mathrm{df}=2, \mathrm{P}=0.7677$; Saprotroph, $\chi 2==3.3885$, $\mathrm{df}=2, \mathrm{P}=0.1837$; SaprotrophPathotroph-Symbiotroph, $\chi 2=1.4926, \mathrm{df}=2, \mathrm{P}=0.4741$ ). 


\section{Supplementary Tables}

Supplementary Table 1. $\mathrm{pH}$, mineral and organic carbon (Cmin and Corg, respectively) and total nitrogen (Ntot) for the three investigated plots in Slovenian Sub-Mediterranean.

\begin{tabular}{llllll}
\hline Plot & $\mathrm{pH}$ & $\mathrm{pH}$ & $\mathrm{Cmin}(\%)$ & Corg (\%) & $\begin{array}{l}\text { Ntot } \\
(\%)\end{array}$ \\
& $\mathrm{H}_{2} \mathrm{O}$ & $0.01 \mathrm{M} \mathrm{CaCl}_{2}$ & & & 0.64 \\
\hline 1 (flysch) & 6.98 & 6.55 & 0.14 & 9.92 & 0.56 \\
2 (limestone) & 5.70 & 4.85 & 0.00 & 7.87 & 0.37 \\
3 (limestone) & 6.28 & 5.51 & 0.02 & 5.41 & \\
\hline
\end{tabular}

Values were obtained for composite samples from five subsamples was determined according to SIST ISO 10390:2006 (pH), SIST ISO 10694:1996 (Cmin and Corg) and SIST ISO 13878:1999 (Ntot). Carbonates were analyzed according to SIST EN ISO 10693:2014 standard. 
Supplementary Table 2. Mean precipitation, temperature, humidity and solar radiation calculated for 30 days before each sampling campaign.

\begin{tabular}{|c|c|c|c|c|c|c|c|}
\hline $\begin{array}{l}\text { Sampling } \\
\text { date }\end{array}$ & $\begin{array}{l}\text { 30-day period before } \\
\text { sampling }\end{array}$ & $\begin{array}{l}\text { Mean } \\
\mathrm{T} \\
{\left[{ }^{\circ} \mathrm{C}\right]}\end{array}$ & $\begin{array}{l}\text { Max. } \\
\mathrm{T} \\
{\left[{ }^{\circ} \mathrm{C}\right]}\end{array}$ & $\begin{array}{l}\text { Min. } \\
\mathrm{T} \\
{\left[{ }^{\circ} \mathrm{C}\right]}\end{array}$ & $\begin{array}{l}\text { Relative } \\
\text { humidity } \\
{[\%]}\end{array}$ & $\begin{array}{l}\text { Sum } \\
\text { solar } \\
\text { radiation } \\
{[\mathrm{h}]}\end{array}$ & $\begin{array}{l}\text { Sum } \\
\text { precipitation } \\
(\mathrm{mm})\end{array}$ \\
\hline $6 / 22 / 2016$ & 22.5.2016-21.6.2016 & 17.7 & 23.2 & 12.4 & 73.5 & 217.9 & 165.9 \\
\hline $8 / 9 / 2016$ & $9.7 .2016-8.8 .2016$ & 22.8 & 29.4 & 16.4 & 60.9 & 309.1 & 25.3 \\
\hline $11 / 9 / 2016$ & $9.10 .2016-8.11 .2016$ & 10.8 & 15.5 & 7.3 & 78.1 & 129.9 & 241.4 \\
\hline $3 / 28 / 2017$ & 25.2.2017-27.3.2017 & 8.7 & 14.5 & 3.8 & 64.7 & 191.1 & 67.1 \\
\hline $6 / 16 / 2017$ & 16.5.2017-15.6.2017 & 19.3 & 25.9 & 12.5 & 62.5 & 324.6 & 35.4 \\
\hline $8 / 22 / 2017$ & 22.7.2017-21.8.2017 & 23.2 & 30.7 & 16.4 & 60.3 & 321.1 & 77.9 \\
\hline $10 / 27 / 2017$ & 26.9.2017-26.10.2017 & 12.8 & 19.6 & 8.0 & 78.2 & 182.0 & 59.1 \\
\hline $11 / 28 / 2017$ & $28.10 .2017-27.11 .2017$ & 8.2 & 12.8 & 4.8 & 75.2 & 97.5 & 151.7 \\
\hline $1 / 30 / 2018$ & $30.12 .2017-29.1 .2018$ & 5.6 & 9.5 & 1.9 & 83.3 & 81.1 & 92.9 \\
\hline $3 / 28 / 2018$ & 25.2.2018-27.3.2018 & 3.4 & 7.3 & 0.3 & 74.8 & 117.8 & 115.4 \\
\hline $5 / 30 / 2018$ & 29.4.2018-29.5.2018 & 17.5 & 23.6 & 12.6 & 73.2 & 204.4 & 123.0 \\
\hline
\end{tabular}


Supplementary Table 3. Results of 'envfit' analysis of environmental variables against NMDS scores for ECM root tip fungal communities.

\begin{tabular}{lll}
\hline Environmental variable & $\mathrm{R}^{2}$ value & $P$-value \\
\hline Mean temperature & 0.0080 & 0.9098 \\
Max temperature & 0.0068 & 0.9210 \\
Min temperature & 0.0087 & 0.9018 \\
Relative humidity & 0.0025 & 0.9674 \\
Solar radiation & 0.0048 & 0.9432 \\
Precipitation & 0.0002 & 0.9979 \\
Mean soil matrix potential & 0.0729 & 0.3875 \\
Min soil matrix potential & 0.0665 & 0.4201 \\
Max soil matrix potential & 0.0527 & 0.5030 \\
Mean soil temperature & 0.0080 & 0.9075 \\
Min soil temperature & 0.0045 & 0.9465 \\
Max soil temperature & 0.0191 & 0.7944 \\
\hline
\end{tabular}


Supplementary Table 4. Root tip fungi revealed to be significantly associated with one or more of the three plots as revealed by indicator species analysis. Values presented are mean relative abundance \pm standard error for cluster one and cluster two. The indicator species statistic and $\mathrm{BH}$-corrected $P$-value are also presented. Bold relative abundance values with an "*" indicate that the taxon was more associated with a plot.

\begin{tabular}{|c|c|c|c|c|c|}
\hline Taxon & $\begin{array}{l}\text { Plot } 1 \\
\text { (Flysch) }\end{array}$ & $\begin{array}{l}\text { Plot } 2 \\
\text { (Limestone) }\end{array}$ & $\begin{array}{l}\text { Plot } 3 \\
\text { (Limestone) }\end{array}$ & Stat & $P$-value \\
\hline Morph 36; Fungal sp. & $0.02 \pm 0.01$ & $0.11 \pm 0.03 *$ & $\begin{array}{l}0.0009 \pm \\
0.0009\end{array}$ & 0.45 & 0.008 \\
\hline $\begin{array}{l}\text { Morph 1; Cenococcum } \\
\text { geophilum }\end{array}$ & $0.05 \pm 0.02$ & $0.22 \pm 0.03 *$ & $0.16 \pm 0.04 *$ & 0.76 & 0.004 \\
\hline $\begin{array}{l}\text { Morph 52; } \\
\text { Agaricomycetes sp. }\end{array}$ & $0.00 \pm 0.00$ & $0.03 \pm 0.02 *$ & $0.00 \pm 0.00$ & 0.36 & 0.016 \\
\hline $\begin{array}{l}\text { Morph 46; Inocybe } \\
\text { splendens }\end{array}$ & $0.002 \pm 0.002$ & $0.00 \pm 0.00$ & $0.06 \pm 0.03 *$ & 0.37 & 0.035 \\
\hline $\begin{array}{l}\text { Morph 6; Sebacina } \\
\text { incrustans }\end{array}$ & $0.26 \pm 0.05 *$ & $0.02 \pm 0.01$ & $0.16 \pm 0.04 *$ & 0.60 & 0.004 \\
\hline $\begin{array}{l}\text { Morph 33; Tomentella } \\
\text { bryophila }\end{array}$ & $0.00 \pm 0.00$ & $0.00 \pm 0.00$ & $0.06 \pm 0.03 *$ & 0.38 & 0.011 \\
\hline
\end{tabular}


Supplementary Table 5. Mycelium ECM fungi revealed to be significantly associated with one or more of the three plots as revealed by indicator species analysis. Values presented are mean relative abundance \pm standard error for cluster one and cluster two. The indicator species statistic and BH-corrected $P$-value are also presented. Bold relative abundance values with an "*" indicate that the taxon was more associated with a plot.

\begin{tabular}{|c|c|c|c|c|c|}
\hline Taxon & Plot 1 (Flysch) & $\begin{array}{l}\text { Plot } 2 \\
\text { (limestone) }\end{array}$ & $\begin{array}{l}\text { Plot } 3 \\
\text { (limestone) }\end{array}$ & $\begin{array}{l}\text { Indicator } \\
\text { statistic }\end{array}$ & $P$-value \\
\hline Sebacina sp. & $0.00 \pm 0.0012$ & $0.00 \pm 0.00$ & $0.00015 \pm 0.00 *$ & 0.80 & 0.002 \\
\hline Pseudotomentella sp. & $0.14 \pm 0.05 *$ & $0.00 \pm 0.00$ & $0.26 \pm 0.08 *$ & 0.88 & 0.002 \\
\hline Sebacina sp. 1 & $0.26 \pm 0.08 *$ & $0.00 \pm 0.00$ & $\begin{array}{l}0.00032 \pm \\
0.00024\end{array}$ & 0.91 & 0.002 \\
\hline Tomentella coerulea & $0.11 \pm 0.05 *$ & $0.09 \pm 0.05 *$ & $0.00 \pm 0.00$ & 0.83 & 0.004 \\
\hline Tomentella punicea & $0.00 \pm 0.00$ & $0.25 \pm 0.08 *$ & $\begin{array}{l}0.00039 \pm \\
0.00026\end{array}$ & 0.74 & 0.004 \\
\hline Amanita pantherina & $0.00 \pm 0.00$ & $0.24 \pm 0.06 *$ & $0.02 \pm 0.01$ & 0.91 & 0.002 \\
\hline $\begin{array}{l}\text { Xerocomus } \\
\text { subtomentosus }\end{array}$ & $0.08 \pm 0.04 *$ & $0.03 \pm 0.01 *$ & $0.0056 \pm 0.0035$ & 0.82 & 0.02 \\
\hline Tomentella bryophila & $0.00 \pm 0.00$ & $0.00 \pm 0.00$ & $0.05 \pm 0.02 *$ & 0.78 & 0.002 \\
\hline Geopora cervina & $\begin{array}{l}7.34 \mathrm{e}-2 \pm 3.51 \mathrm{e}- \\
2 *\end{array}$ & $0.00 \pm 0.00$ & $\begin{array}{l}2.20 \mathrm{e}-5 \pm 2.20 \mathrm{e}- \\
5\end{array}$ & 0.59 & 0.02 \\
\hline Fungal sp. & $0.00 \pm 0.00$ & $0.00 \pm 0.00$ & $0.10 \pm 0.05 *$ & 0.74 & 0.002 \\
\hline Tomentella pilosa & $0.00 \pm 0.00$ & $0.00 \pm 0.00$ & $\mathbf{0 . 0 7} \pm \mathbf{0 . 0 3}$ & 0.67 & 0.004 \\
\hline $\begin{array}{l}\text { Trichophaea } \\
\text { woolhopeia }\end{array}$ & $0.00 \pm 0.00$ & $0.00 \pm 0.00$ & $0.04 \pm 0.02 *$ & 0.67 & 0.004 \\
\hline Fungal sp. 1 & $0.00 \pm 0.00$ & $0.00 \pm 0.00$ & $0.02 \pm 0.01 *$ & 0.62 & 0.007 \\
\hline Cortinarius sp. & $0.05 \pm 0.03$ & $0.00 \pm 0.00$ & $0.00 \pm 000$ & 0.54 & 0.02 \\
\hline Tomentella sp. 1 & $\begin{array}{l}3.34 \mathrm{e}-5 \pm 3.34 \mathrm{e}- \\
5\end{array}$ & $\begin{array}{l}3.42 \mathrm{e}-2 \pm 3.44 \mathrm{e}- \\
2 *\end{array}$ & $0.00 \pm 0.00$ & 0.62 & 0.01 \\
\hline $\begin{array}{l}\text { Tomentella } \\
\text { umbrinospora }\end{array}$ & $\begin{array}{l}2.51 \mathrm{e}-2 \pm 1.01 \mathrm{e}- \\
2 *\end{array}$ & $0.00 \pm 0.00$ & $\begin{array}{l}8.43 \mathrm{e}-5 \pm 6.81 \mathrm{e}- \\
5\end{array}$ & 0.77 & 0.004 \\
\hline Inocybe luteifolia & $0.02 \pm 0.01 *$ & $0.0014 \pm 0.0014$ & $0.00 \pm 0.00$ & 0.62 & 0.006 \\
\hline Lactarius quietus & $\begin{array}{l}6.64 e-3 \pm 3.53 e- \\
3 *\end{array}$ & $\begin{array}{l}2.55 \mathrm{e}-5 \pm 2.55 \mathrm{e}- \\
5\end{array}$ & $0.00 \pm 0.00$ & 0.80 & 0.002 \\
\hline
\end{tabular}




\begin{tabular}{llllll} 
Sebacina sp. 2 & $\mathbf{0 . 0 0 3 6} \pm \mathbf{0 . 0 0 1 2}$ & $0.00 \pm 0.00$ & $0.00 \pm 0.00$ & 0.73 & 0.002 \\
& $*$ & & & \\
Inocybe asterospora & $0.00 \pm 0.00$ & $\begin{array}{l}\mathbf{0 . 0 0 9 8} \pm \mathbf{0 . 0 0 8 2} \\
*\end{array}$ & $0.00 \pm 0.00$ & 0.58 & 0.02 \\
Tomentella sp. 5 & $0.00011 \pm$ & $\mathbf{0 . 0 0 3 0} \pm \mathbf{0 . 0 0 1 2}$ & $0.00 \pm 0.00$ & 0.61 & 0.03 \\
& 0.000060 & $*$ & & \\
Tomentella punicea 2 & $0.00 \pm 0.00$ & $\mathbf{0 . 0 0 2 2} \pm \mathbf{0 . 0 0 0 8 3}$ & $0.00 \pm 0.00$ & 0.62 & 0.008 \\
& & $*$ & & & \\
Tomentella sp. 20 & $0.000027 \pm$ & $\mathbf{0 . 0 0 0 5 2} \pm$ & $0.00 \pm 0.00$ & 0.56 & 0.03 \\
& 0.000027 & $\mathbf{0 . 0 0 0 2 6} *$ & & & \\
\hline
\end{tabular}




\section{Supplementary Figures}

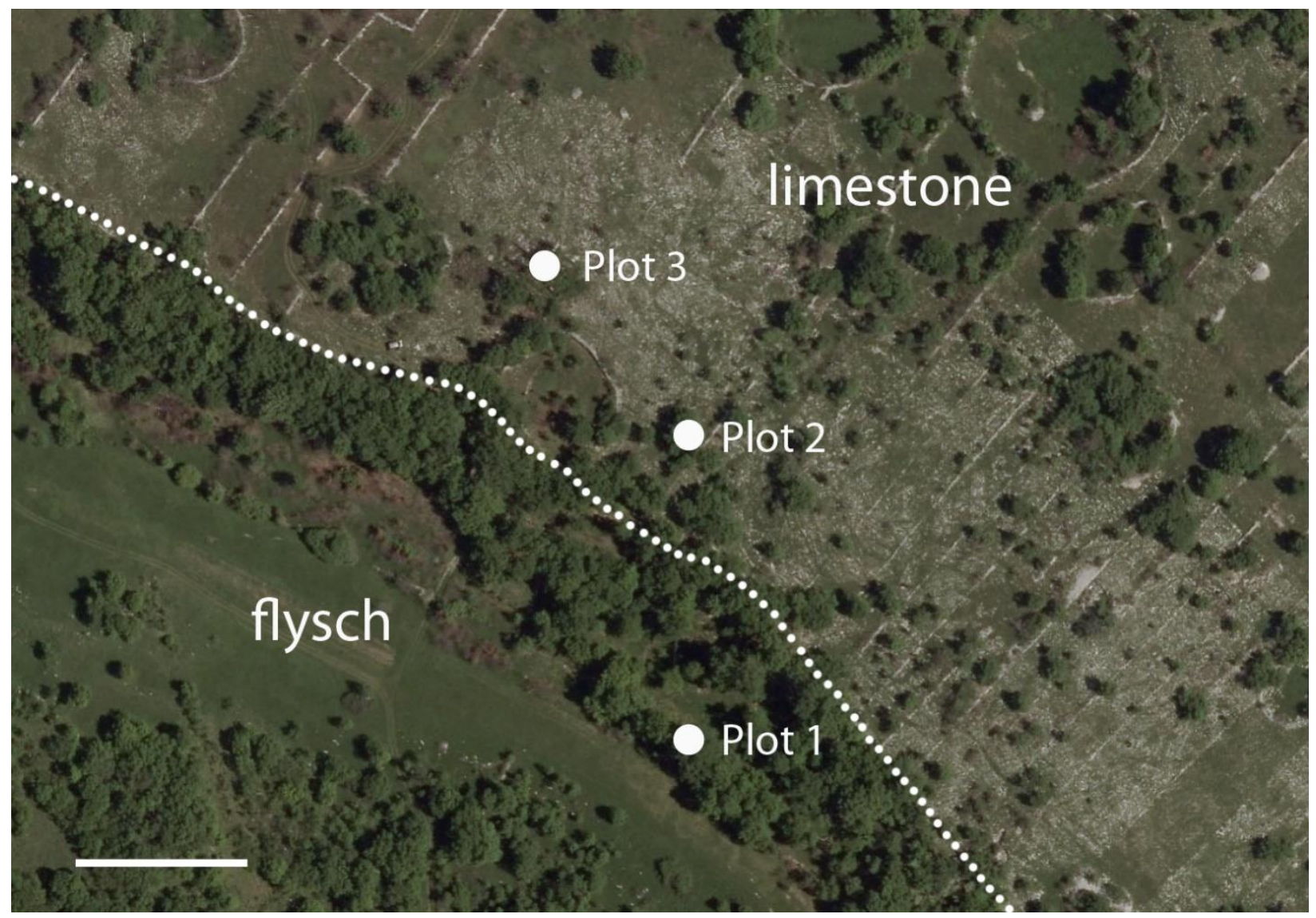

Supplementary Figure 1. Sampling plots for ECM root tips of $Q$. pubescens and soil mycelium fungi at the field site within the Slovenian Sub-Mediterranean. Limestone and flysch ecotypes are separated by the dotted line. The scale bar in the lower left corner represents $50 \mathrm{~m}$ distance. Plot 1 was located on flysch bedrock, whereas plots 2 and 3 were located on limestone bedrock. Aerial photo by ARSO (http://gis.arso.gov.si/atlasokolja, 9.7.2020). 


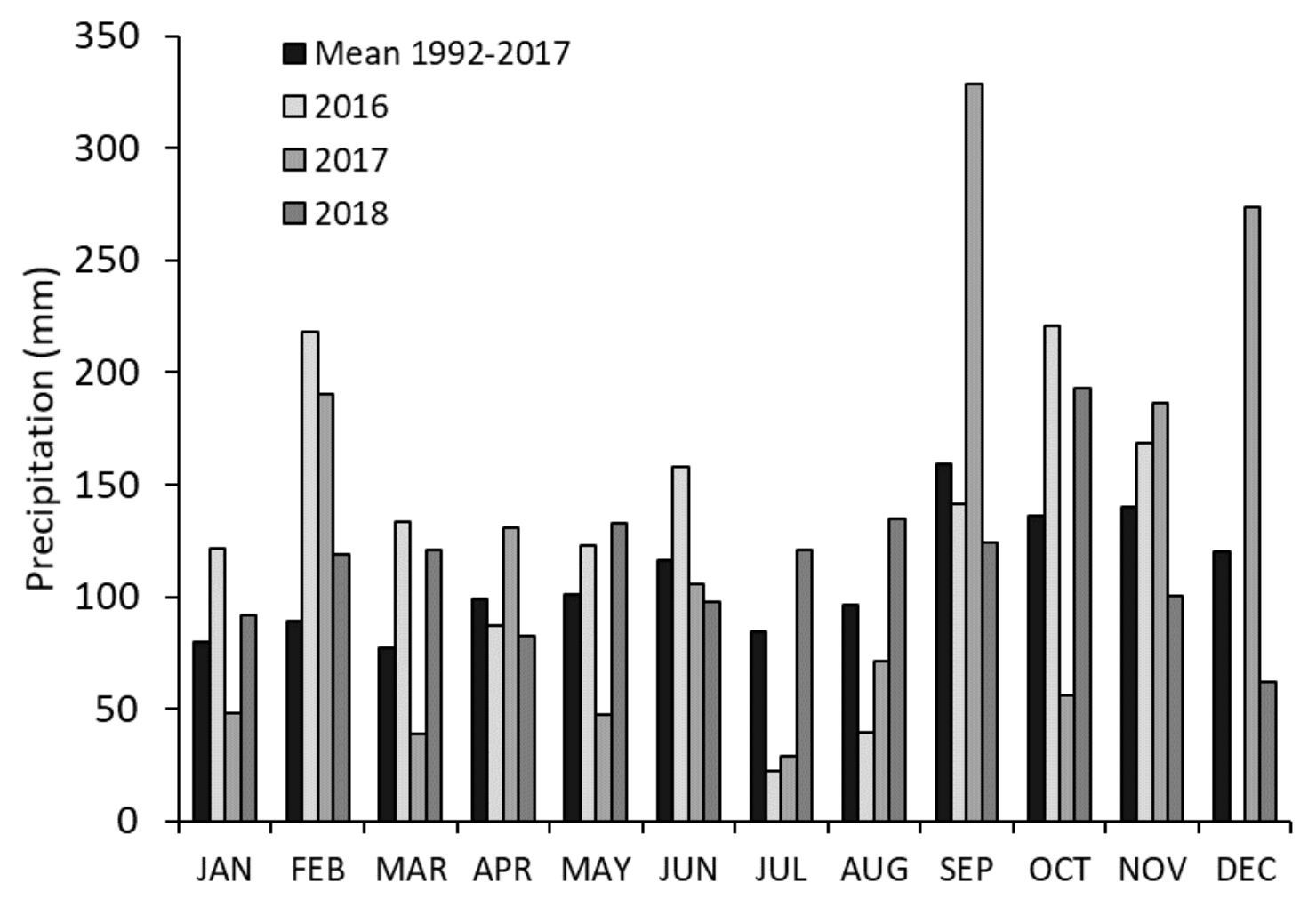

Supplementary Figure 2. Monthly precipitation at the field site throughout the sampling period (June 2016-May 2018) compared to the 25-year average (1992-2017). 

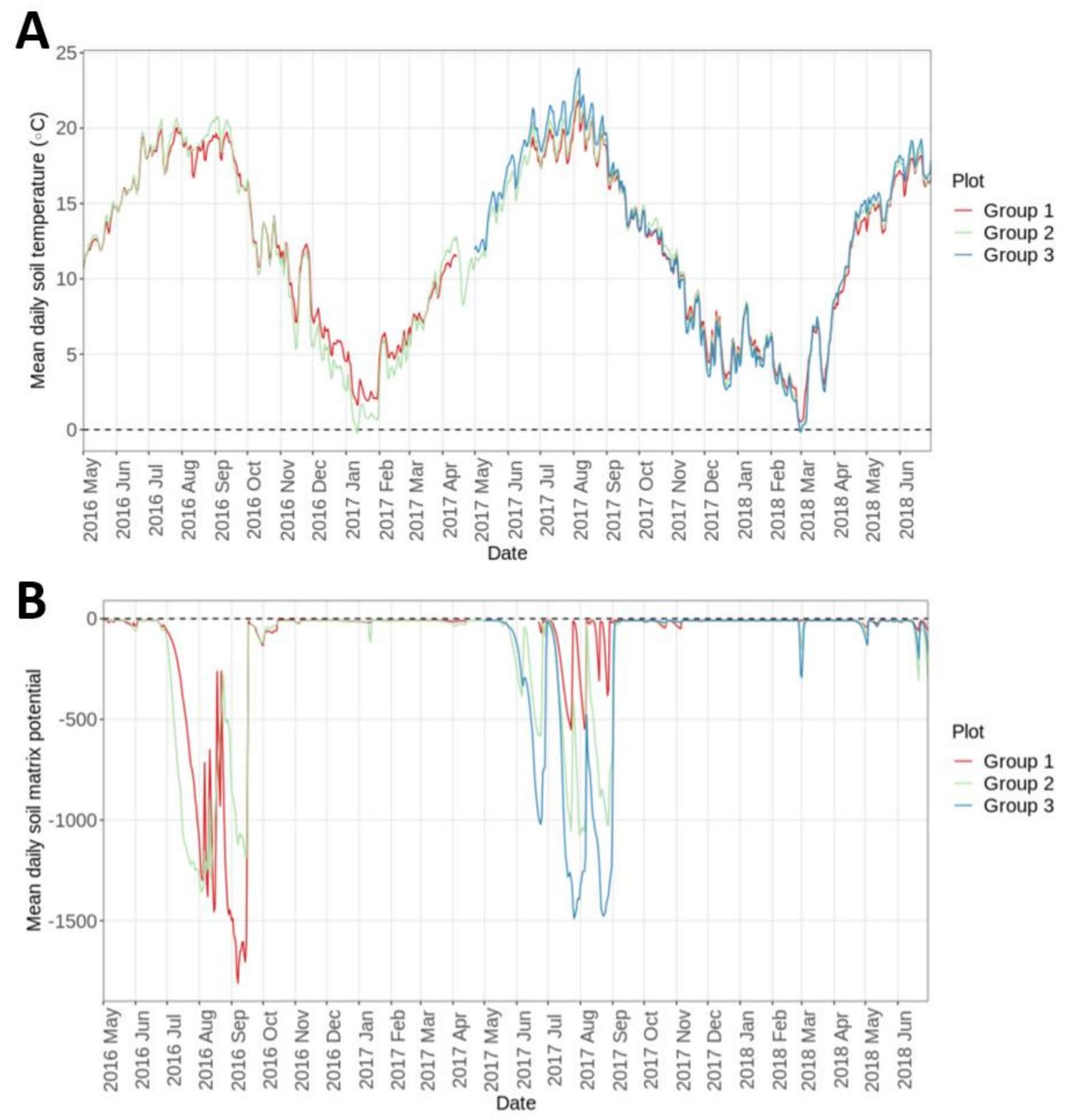

Supplementary Figure 3: Daily averages for soil temperature (a) and soil matrix potential (b) at the three Quercus pubescens sites in Slovenian Sub-Mediterranean measured using MPS-2 dielectric water potential sensors (Decagon devices Inc., USA) at 10-minute intervals from May 2016 till June 2018. 


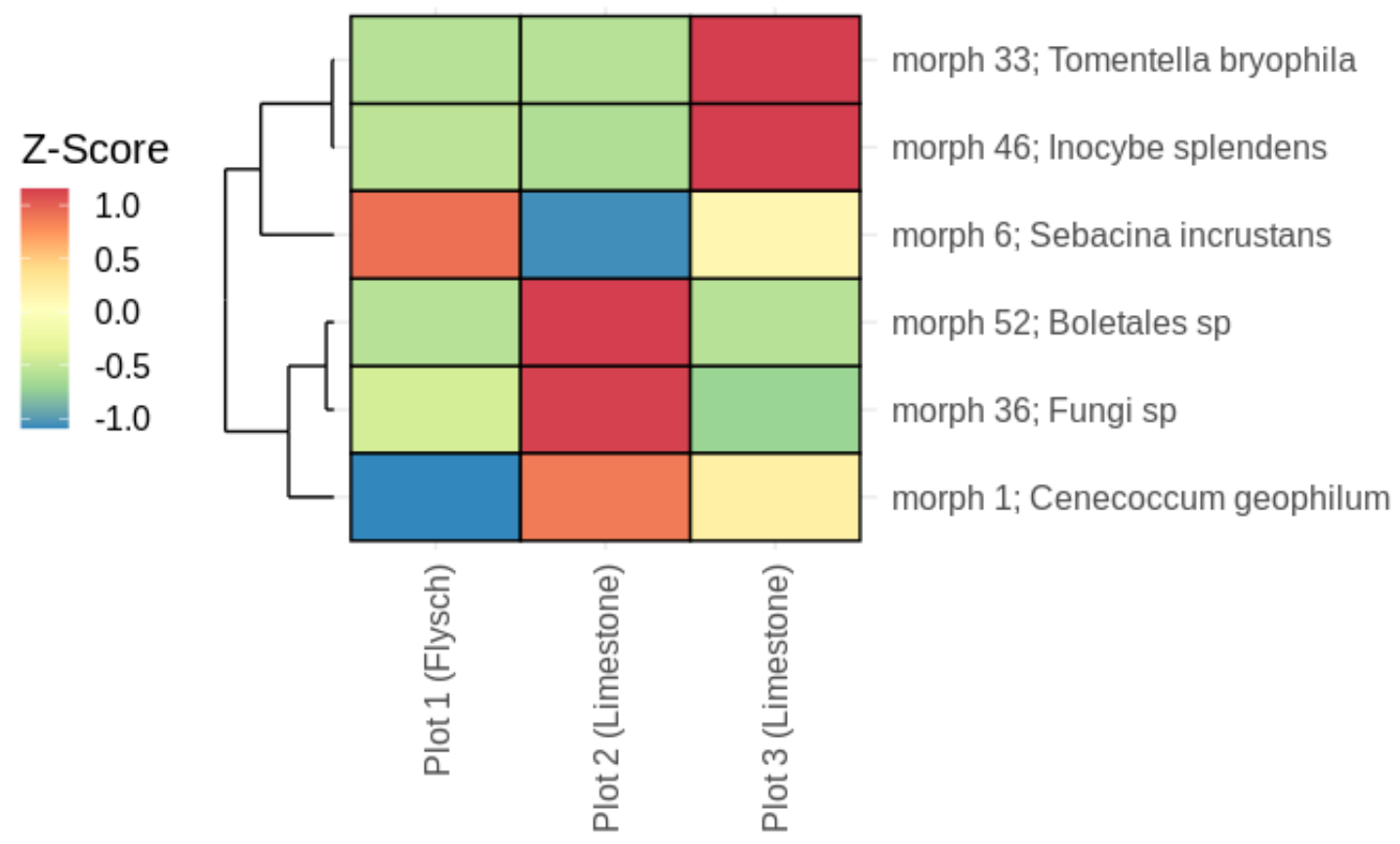

Supplementary Figure 4. Heatmap of Q.pubescens root tip ECM fungi revealed to be significantly associated with one or more of the field plots, as revealed by indicator species analysis. Values presented are based on Z-score normalization of OUT abundances by row (species). The dendogram shows the results of hierarchical clustering of Euclidean distances between OTUs using the 'complete' method. 


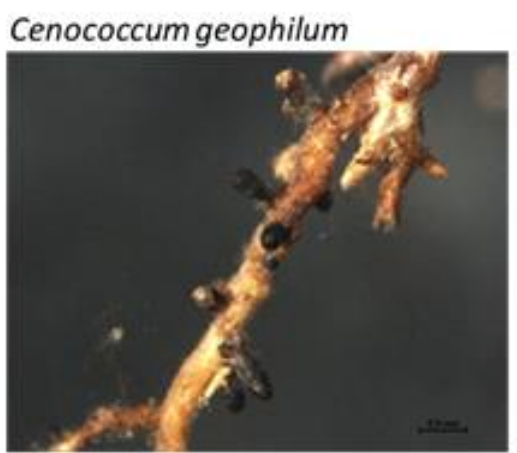

Inocybe splendens
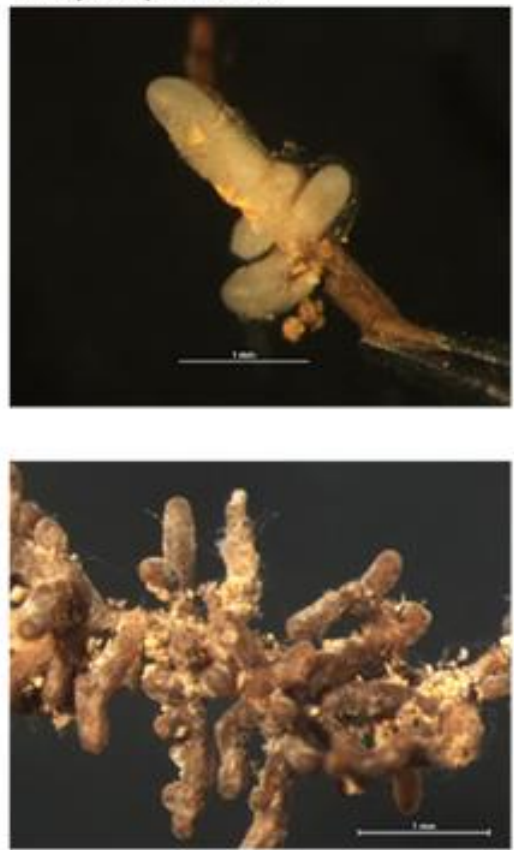

Morphotype 36
Tomentella bryophila
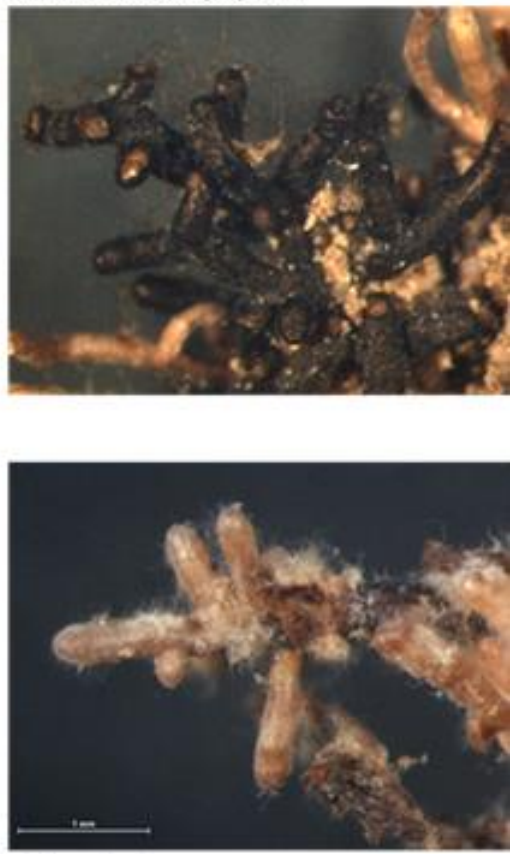

Sebacina incrustans

Supplementary Figure 5: Indicator species with significant associations with one or two Quercus pubescens plots in Slovenian Sub-Mediterranean. 


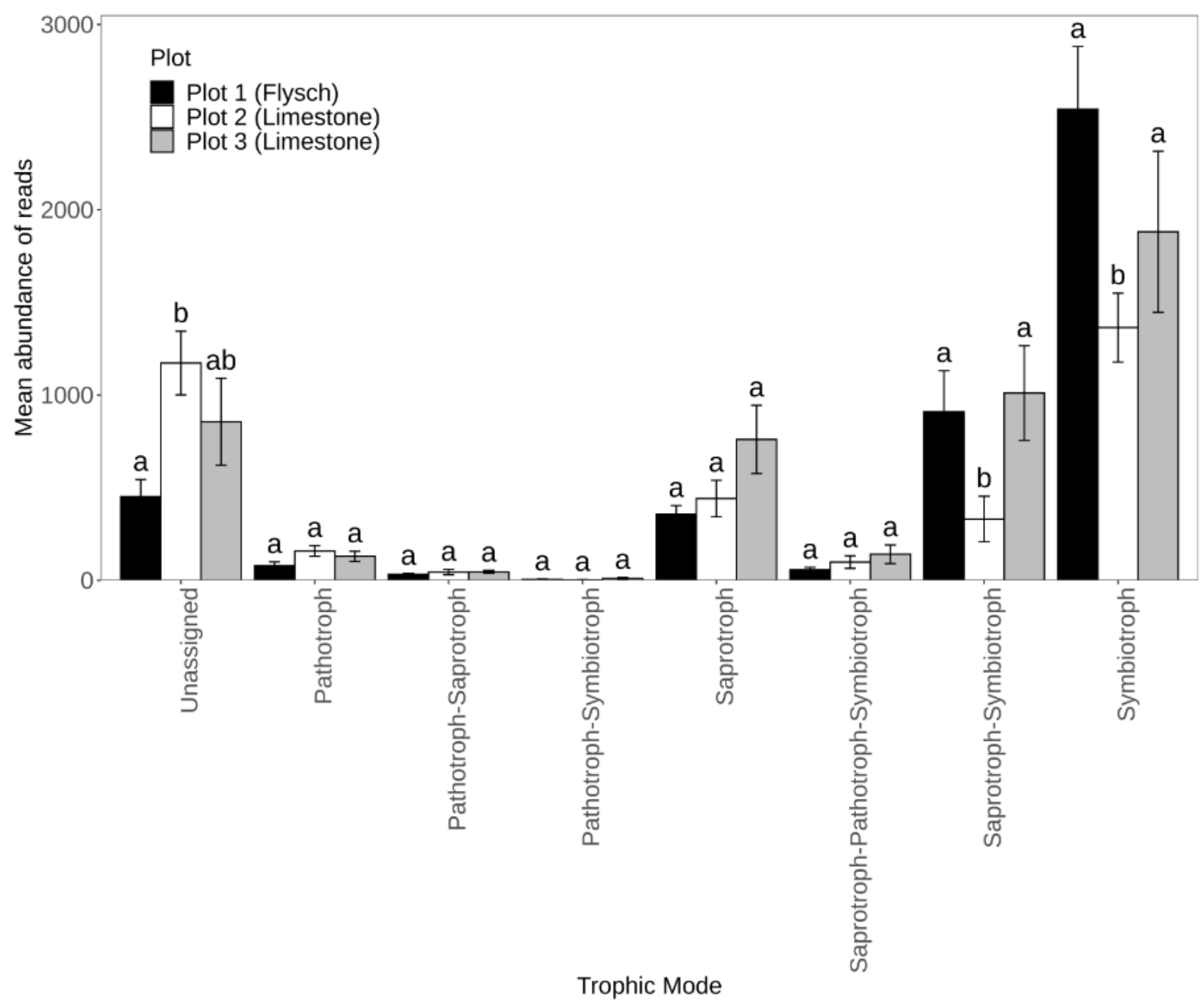

Supplementary Figure 6. Assignment of trophic modes to mycelium in mesh bags at the three $Q$. pubescens plots in the Slovenian Sub-Mediterranean (mean \pm stderr). Significant differences were tested by Kruskal-Wallis and Dunn's post-hoc test and are marked with different letters when $P<0.05$. 


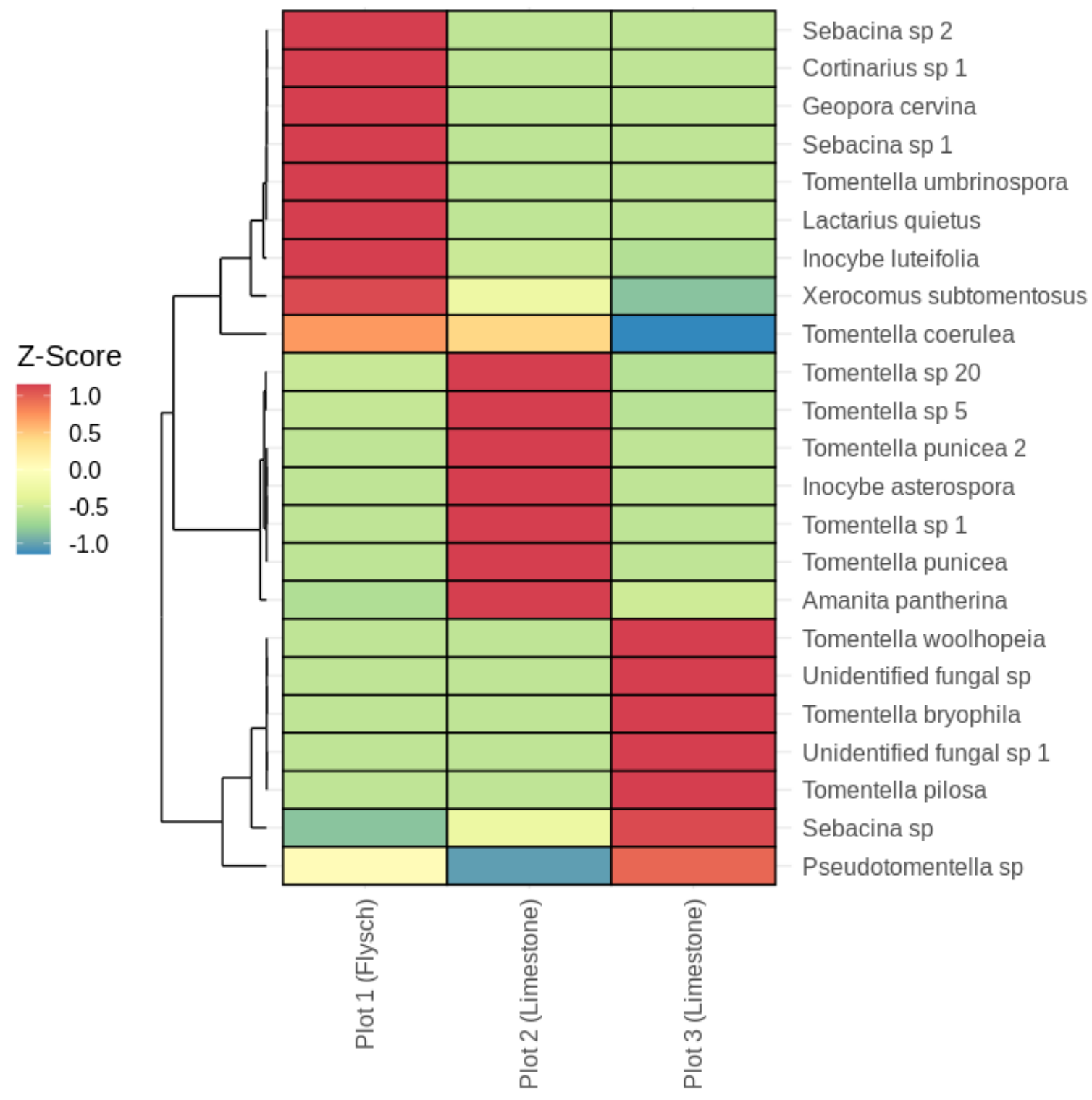

Supplementary Figure 7. Heatmap of ECM mycelium fungi revealed to be significantly associated with one of more field plots, as revealed by indicator species analysis. Values presented are based on Z-score normalization of OUT abundances by row. The dendrogram shows the results of hierarchical clustering of Euclidean distances between OTUs using the 'complete' method. 